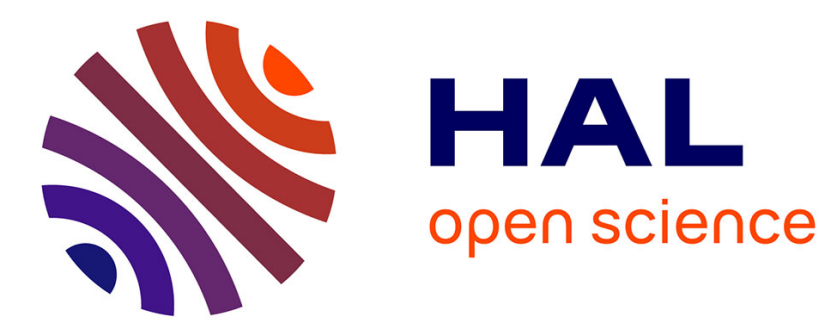

\title{
An Information Theory Perspective for the Binary STT-MRAM Cell Operation Channel
}

\author{
Jianxiao Yang, Benoit Geller, Meng Li, Tong Zhang
}

\section{To cite this version:}

Jianxiao Yang, Benoit Geller, Meng Li, Tong Zhang. An Information Theory Perspective for the Binary STT-MRAM Cell Operation Channel. IEEE Transactions on Very Large Scale Integration

(VLSI) Systems, 2016, 10.1109/TVLSI.2015.2436370 . hal-01224232

\section{HAL Id: hal-01224232}

\section{https://hal-ensta-paris.archives-ouvertes.fr/hal-01224232}

Submitted on 15 Nov 2015

HAL is a multi-disciplinary open access archive for the deposit and dissemination of scientific research documents, whether they are published or not. The documents may come from teaching and research institutions in France or abroad, or from public or private research centers.
L'archive ouverte pluridisciplinaire HAL, est destinée au dépôt et à la diffusion de documents scientifiques de niveau recherche, publiés ou non, émanant des établissements d'enseignement et de recherche français ou étrangers, des laboratoires publics ou privés.

\section{(1)(1) $\$(0)$}

Distributed under a Creative Commons Attribution - NonCommercial - ShareAlikel 4.0 


\title{
An Information Theory Perspective for the Binary STT-MRAM Cell Operation Channel
}

\author{
Jianxiao $\mathrm{YANG}^{1}$, BenoitGELLER ${ }^{1}, \mathrm{Meng}^{\mathrm{L}}{ }^{2}$, and Tong $\mathrm{ZHANG}^{3}$
}

\begin{abstract}
Spin-torque transfer magnetic random access memory (STT-MRAM) has emerged as a promising non-volatile memory technology, with advantages such asscalability, speed, endurance and power consumption. This paper presents a STT-MRAM cell operation channel model with write and read operations for information theorists and error correction code designers. This model takes into account the effects of process variations and thermal fluctuations and considers all principle flaws during the fabrication and operation processes. With this model, evaluations are not only made for the write channel, the read channel, but alsothe write and read channel with metricssuch as operation failure rate, bit error rate, channel ergodiccapacity and channel outage probability at certain outage capacity. Moreover, it is proved that the distributions of written-in bit states are not uniformly distributed andare proportional to their respective write success probabilities. Finally, simulation results show that practical code rates and code block lengths can guarantee reliable performances only if the operation success rate difference between state ' 1 ' and state ' 0 ' is small enough.
\end{abstract}

Index Terms-Spin-Torque Transfer Magnetic Random Access Memory (STT-MRAM), Non-Volatile Memory (NVM), Error Correction Code (ECC), Write Operation, Read Operation, Channel Model, Operation Failure Rate, Bit ErrorRate (BER), Channel Ergodic Capacity, Channel Outage Capacity, Channel Outage Probability.

\section{INTRODUCTION}

$\mathrm{S}$ PIN-TRANSFERtorque magnetic random access memory (STT-MRAM) has become one of the most promising candidate for nextgeneration memory in terms of high-speed, nonvolatility and ultra low-power consumption. All these features make STT-MRAMextremely attractive to become general memories (cache, main memory, disk) for mobile devices.

However, STT-MRAM suffers from process variations such as MOS transistor device variations, magnetic tunneling junction (MTJ) geometry variations, and resistance variations,

Manuscript received This work was supported in part by the ANR Greencocom project.

${ }^{1}$ Jianxiao YANG and Benoit GELLER are with Department U2IS of ENSTA-ParisTech, Université Paris SAclay, 828 Boulevard des Maréchaux, 91120, Palaiseau, France (e-mail: jianxiao.yang, benoit.geller@ensta-paristech.fr);

${ }^{2}$ Meng LI is with Green Radio Group of IMEC, Kapeldreef 75 , 3001,Heverlee, Belgium(e-mail: meng.li@imec.be);

${ }^{3}$ Tong ZHANG is with the Department of Electrical, Computer, and Systems Engineering, Rensselaer Polytechnic Institute, Troy, NY 12180-3590, USA (e-mail:tong.zhang@ieee.org). which become even more critical as the technology scales down. Moreover, as many electronic devices, STT-MRAM suffers from random thermal fluctuations which are independent of the device process variations.Therefore, complementary to making a difficult cell optimization among different technical parameters mutually correlated, it is possible to reduce the design complexity and tolerate a certain level of device imperfection by introducing another degree-of-freedom, i.e., error correction codes (ECC) [1],[2].

In order to design asatisfying ECC, the STT-MRAM cell channel needs to be carefully modeled and investigatedby obtaining metrics such asoperation failure rate, bit error rate (BER), channel ergodiccapacity and channel outage probability at certain outage capacity. This model is extremely important for the efficient selection ofboth the code rate and the code lengthin order to meet the practical performance requirements.The scientific canvas for this design is information theory; STT-MRAM is then considered as a device having an input (i.e., the original information) and an output (i.e., a resistance corresponding to the written/read out information), the output being statistically linked to the input through the physical properties of the media. For more distorted channels, more redundancy should be added by the ECC, i.e. the code rate between the real informationand the coded bits (information and added redundancy) should be lower and Shannon postulated that an asymptotically small error can be achieved if the code rate is less than the channel capacity[3]. However, there are very few works correlating the ECC design with the cell channel.[4] modeled the STT-MRAM operation channel as an asymmetrical resistance variation channel; both the influence ofthe write and read failures, and the process variations are considered as factors enlarging the standard deviations of resistance distributions which is over-simplifying as the write and read failures are nonlinear processes.Moreover, [5]proposed an asymmetric write channel model taking into account process variations and thermal fluctuations. However, in a STT-MRAM memory system, the write and the readoperations are both important and each of these functions must be modeled carefully; actually the optimization on either the write or the read operation generally does not lead to a global optimization and technical parameters are usually selected to balanceboth write and read performances to reach a global optimum performance.Taking the transistor width as an example, the selection of the transistor width generally determinesthe current drive capacity;on one side,the write operation needs a large current to target the magnetization as 
quickly as possible, andthe read operationrequires a sufficient current to drive the sense amplifier as fast as possible; on the other side,the read current should be kept small enough to avoid flipping the cell content.Differently from [4][5], [6] proposed an optimization technique to minimize both read and write failures and developed a mixed-mode framework to optimize the bit-cell level reliability. This framework captured the transport physics by using the non-equilibrium Green's function method, solved the MTJ magnetization dynamics with the Landau-Lifshitz-Gilbert (LLG) equation, and performed bit-level optimization with HSPICE. [7]dealt with the reliability issues by analyzing the impact of the nonpermanent "soft-errors"introduced by various operations,as well as thepermanent "hard-errors" caused by permanent device damages.[6] and[7]concentrate on hardware design in order to optimize the memory performance.However, such a performance level can also be reached by using an additional degree-of-freedom - a properly designed ECC[1],[8]-[13]; the hardware design complexity can thus tolerate a certain level of unreliability that will be improved by ECC.

Differently fromthe previous works[4]-[7], this paper intends to propose a channel model to simulatethe reliability of the basic STT-MRAM cells write and read operationsby taking into account both the process variations and thermal fluctuations(without considering the impact of "hard-errors");also differently from the compact models [14]-[18], this model targets to bridge the gap between the information theory community and the physical device community by taking into account various process variations and thermal fluctuations without solving any complex equations.Moreover, aiming at an efficient ECC design, operation failure rates, bit error rates and channel capacitiesareevaluated.Comments are also made for the highly asymmetrical characteristics of the STT-MRAM channel. Finally, suggestions are made for the selection of both the code rate and the code block length.

The rest of the paper is organized as follows. The basics of STT-MRAM cell operations, various process variations, thermal fluctuations and capacity definitions arebriefly reviewed in Section II. The proposed channel model including both write and read operations isdetailed in Section III. Simulation and numerical results are given in Section IVwith comments on ECC design. Finally, conclusions are made in Section V.

\section{STT-MRAM CELLOPERATIONS}

\section{A. STT-MRAM Cell Basics}

A datum in a STT-MRAM cell is represented as the resistance state ofa magnetic tunneling junction (MTJ) device, which can be switched by applying programming currents with different polarizations[19],[20]. A widely used STT-MRAM cell structure is displayed in Fig.1 and the so-called "1T-1MTJ" structure consists of one transistor and one magnetic tunneling junction, where a tunneling oxide layer (see the grey bars in Fig.1) is sandwiched between two ferromagnetic layers; one of these layer is called reference layer (RL) and has a fixed magnetization and the other layer is called free layer (RL) with two possible magnetizations to represent a bit.
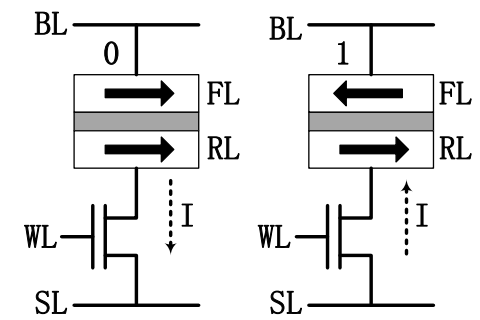

Fig.1. STT-MRAM 1T-1MTJ cell structure: (a) bit "0".(b) bit "1".

Writing a "0" or writing a "1" to a cell is achieved by applying reversed direction currents. When writing "0" (MTJ in parallel state), the word line (WL) and bit line (BL) are connected to the supply voltage $V_{\mathrm{DD}}$, and the source line (SL) is connected to the ground (see Fig.1.(a)). The NMOS transistor is either working in its saturation regionfor a small transistor width or in its linear region for a large transistor width. When writing "1" (MTJ in anti-parallel state), WL and SL are connected to $V_{\mathrm{DD}}$, while $\mathrm{BL}$ is connected to the ground (see Fig.1.(b)). The transistor is then working in its saturation region.

There are two ways to read a cell, the so-called parallel (P) direction read with the same direction as writing " 0 ", and the anti-parallel (AP) read with the same direction as writing "1". Inthe parallel direction reading, a low voltage is applied between BL and SL. After activating WL, a current flows from BL to SL.In the anti-parallel direction reading, the voltage polarity applied to BL and SL is switched and a current flows in the reversed direction - from SL to BL.

\section{B. CMOS Process Variations}

The CMOS process variations contribute to the variability of the driving strength of the NMOS transistor due to random dopantfluctuations, line-edge roughness, shallow trench isolationstress, and geometry variations of the transistor channel length/width[21]. All these process variations have a direct impact over the transistor's threshold voltage $V_{\text {TH }}$ and its equivalent resistance.

\section{MTJ Process Variations}

The MTJ process variationsare independent from the CMOS process variations and lead to the variability of the MTJ. Thesevariationsstem from the MTJ shaping variations, from the oxide thickness variation, and from the localized fluctuation of magnetic anisotropy[22]. The first two factors cause thevariations of the MTJ resistance and of the MTJ switching current by changing the bias conditions of the NMOS transistor, whereas the third factor is an intrinsic variation of the magnetic material that both affects the MTJ'scritical switching current density $J_{\mathrm{C} 0}$ and the magnetization stability barrier height.

\section{Random Thermal Fluctuations}

In general, the magnetization dynamics of the MTJ switching affected by thermal fluctuations can be modeled by the famous 
Landau-Lifshitz-Gilbert (LLG)equationby considering thethermal agitation fluctuating field[23].Due to the random thermal fluctuations, the MTJ switching time becomes unrepeatable and is independent of the process variations.

It has been found that switching modes in MTJ are categorized as a function of the switch current duration and can be classified into three distinct modes[24]: thermal activation, dynamic reversal and precessional switching.

For a long current pulse(longer than $10 \mathrm{~ns}$ ), the magnetization switching is a thermally activated process. In this regime, the magnetization switching is independent of the initial conditions and is only determined by thermal agitation during the switching process.

For a very short switch current duration(shorter than $3 \mathrm{~ns}$ ), the magnetization switching is precessional switching and is mainly dependent on the initial thermal distribution. In this regime, both the magnetization switching distribution and the switching probability are independent of the thermal agitation during the switching process.

For an intermediate current pulseduration (between 3 ns and $10 \mathrm{~ns}$ ), the magnetization switching is dynamic reversal[24] and is determined by the initial thermal distribution and bythe thermal agitation during the switching process.

\section{E. Write Variations}

During the write operation, two kinds of failures can occur :

1) The cell fails to be flipped from 0 to 1 and keeps the 0 state while anti-parallel writing is performed;

2) The cell fails to be flipped from 1 to 0 and stays at the 1 state when parallel writing is performed.

These failures come from two factors that can lead to the variation of the MTJ switching current and thus result ona switching time uncertainty [25]: one factor is the CMOS transistor and MTJ process variations, which causea driving ability variation of the transistor; the other factor is therandomthermal fluctuations, inducing a stochastic MTJ magnetization switching process[23].

Moreover, these two factors leadto a high asymmetry between the two writing state transitions $0 \rightarrow 1$ and $1 \rightarrow 0$. The bias difference condition [26] of the transistor causes that the $0 \rightarrow 1$ transition requires a longer time to perform the transition compared to the $1 \rightarrow 0$ transition, and the standard deviation(STD) of the transition $0 \rightarrow 1$ is much broader thanthe one of the transition $1 \rightarrow 0$ [27]. Therefore the write operation $0 \rightarrow 1$ contributes prominently to writing failure events [26] and is considered as an "unfavorable" switching direction.

\section{F. Read Variations}

One must achievea compromise on setting a proper read current[6],[7],[28] for the read operation: on one side, the read current requiresto be high enough to generate a sufficient sense voltage margin to drive the sense amplifier and to ensure a fast read access time; on the other side, the read current must be kept low enough so as to avoid flipping the stored state to the reversed one.

Therefore, three types of errors can occur during the read operation:
1) The cell stores a 0 but is read out as a 1 ;

2) The cell stores a 1 but is read out as a 0 ;

3)The cell stores a 0 (resp. 1) but is flipped to 1 (resp.0) during an anti-parallel (resp.parallel) read operation.

The first two error types come from the process variations of the cell MTJs and transistors, when compared to a reference resistance which is assumed to be ideal with neither process variations nor thermal fluctuations; the third error type stems from too large read current flipping the MTJ cell state.

\section{G. Channel Capacity}

In order to design an efficient ECC with reliable performance for STT-MRAM systems, not only should the operation failure ratesbe measured, but also the operational channel capacity, i.e., the maximum ratio that can be reliably written into and read out from 1T-1MTJ cells, needs to be evaluated.

For the STT-MRAM write and read channel, the capacity can be written as:

$$
C=\max _{\{p(x)\}}\{I(X ; Y)\},
$$

where $X=\{0,1\}$ is the input of the channel, and $Y$ is a continuous output resistance value.

Since the a priori information about the input bit $x$ is highly content dependent, it is reasonable to assume an equiprobable distribution for $x$, i.e., $p(x=0)=p(x=1)=0.5$. Therefore, the channel capacity is equal to the mutual information $I(X ; Y)$, given by:

$$
\begin{aligned}
C & =I(X ; Y) \\
& =H(Y)-H(Y \mid X),
\end{aligned}
$$

where $H(Y)$ is the entropy of the channel output:

$$
H(Y)=-\int p(y) \log _{2}(p(y)) d y
$$

and the probability density function $p(y)$ is:

$$
p(y)=\sum_{x=0}^{1} p(x) p(y \mid x) ;
$$

moreover, $H(Y \mid X)$ is the conditional entropy of the channel output $Y$ given the channel input $X$, defined as:

$$
H(Y \mid X)=-\sum_{x=0}^{1} \int p(y \mid x) p(x) \log _{2}(p(y \mid x)) d y
$$

Note that (2) can be applied to the capacity evaluation of the write channel, the read channel, the write and read channel in order to balance write and read operations.

The capacity (2), also called ergodic capacity, is obtained by averaging overall possible channel realizations (i.e., an infinite number of 1T-1MTJ cells). This implies that the ergodic capacitycan be achieved only by a theoreticinfinite length ECC.

However, in practice, for a finite code length, the channel capacity varies from one block to another due to the limited number of channel realizations. The outage probability $\varepsilon_{o}$ [29]ismore useful in this case; $\varepsilon_{o}$ is defined as the probability that a capacity $C_{N}$ measured over a finite sample of size $N$ is lower than a given capacity threshold $C_{o}$, where $C_{N}$ represents the actual data rate and $C_{o}$ represents a target data rate that is able to be correctlymemorized and delivered. When theactual 
block-wise channel capacity $C_{N}$ is smaller than the required data rate $C_{o}$, no ECC exists to guarantee a zero errorevent and a decoding failure isthus declared. In other words, if a design target with a block code of length $N$ bits and a decoding failure rate $\varepsilon_{o}$ are set, the maximum useful information bit number is $N C_{N}$ and the minimum redundant bit numberintroduced by the ECC should be $N\left(1-C_{N}\right)$. Mathematically, the definition of the outage probability is given by:

$$
\operatorname{Pr}\left(C_{N}<C_{o}\right) \square \varepsilon_{o},
$$

where the terms $C_{N}, H\left(X_{N}\right)$ and $H\left(X_{N} \mid y_{n}\right)$ can be computed as follows:

$$
\begin{gathered}
C_{N}=\frac{1}{N} \sum_{n=1}^{N}\left(H\left(X_{n}\right)-H\left(X_{n} \mid y_{n}\right)\right), \\
H\left(X_{n}\right)=-\sum_{x_{n}=0}^{1} p\left(x_{n}\right) \log _{2}\left(p\left(x_{n}\right)\right), \\
H\left(X_{n} \mid y_{n}\right)=-\sum_{x_{n}=0}^{1} p\left(x_{n} \mid y_{n}\right) \log _{2}\left(p\left(x_{n} \mid y_{n}\right)\right), \\
p\left(x_{n} \mid y_{n}\right)=\frac{p\left(x_{n}\right) p\left(y_{n} \mid x_{n}\right)}{\sum_{x=0}^{1} p(x) p\left(y_{n} \mid x\right)} .
\end{gathered}
$$

It is noted that $y_{n}$ is just onerealizationof $Y$ anda finite block of $N$ realizations cannotcover the whole distribution of $Y$.

\section{STT-MRAM CELL OPERATION CHANNEL}

In this section, a complete STT-MRAM cell operation channel model with both write and read operations is proposed. This model considerstransistor and MTJ process variations, random thermal fluctuations, writing failures, reading flipping errors and resistance variations.
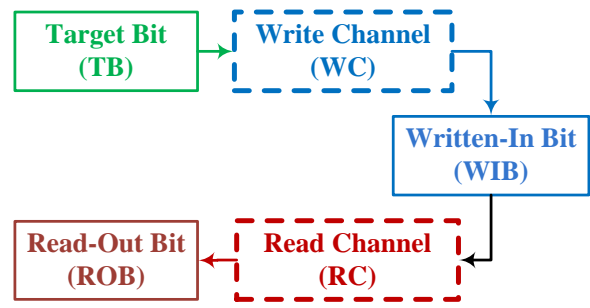

Fig.2. Block diagram of STT-MRAM operation channel model.

The proposed complete cell channel model is shown in Fig.2 and includestwo operationsand three states. The two operations - write channel and read channel are further elaborated inFig.3 and Fig.6, respectively. The three states - target bit (TB), written-in bit (WIB) and read-out bit (ROB) represent the three different livingstates where a bit message resides respectively before writing, after writing (or before reading), and after reading.

\section{A. WriteOperation Channel}

The write operation channel model is divided into 5 consecutivesteps:

1) Generate the mean write current value $\bar{I}_{w}^{P V}$ for the target bit $w=\{0,1\}$;
2) Add a random variation to the mean write current $\bar{I}_{w}^{P V}$ to generatetheaffectedwritecurrent $I_{w}^{P V}$ processvariation;

3) Map theprocess variation affected switching current $I_{w}^{P V}$ to a flipping time $T_{w}^{P V}$;

4) Generate thefinal switching time $T_{w}^{F S T}$ by further introducing a random thermal-induced deviation $\Delta T_{w}^{T F}$ to the previous flipping time $T_{w}^{P V}$ :

$$
T_{w}^{F S T}=T_{w}^{P V}+\Delta T_{w}^{T F}
$$

5) Finally, a writing operation success/failure decision is made by comparing the given write pulse duration (WPD) $T_{w}^{W P D}$ with the required final switchingtime $T_{w}^{F S T}$

. If $T_{w}^{W P D} \geq T_{w}^{F S T}$, the written-in bit $z$ is successfully updated asthe target bit $w$; otherwise, the write operation fails and the WIB $z$ keeps the previous state $z^{-}$before this write operation, i.e.,

$$
z=\left\{\begin{array}{cc}
w, & \text { if } T_{w}^{W P D} \geq T_{w}^{F S T} \\
z^{-}, & \text {else }
\end{array}\right.
$$

Moreover, the soft WIB state $z$, i.e., the MTJ resistance value $R_{\mathrm{Z}}$ [22],[25], is such that:

$$
R_{Z} \propto \exp \left(t_{\mathrm{ox}}\right) / A_{\mathrm{MTJ}},
$$

where $t_{\mathrm{ox}}$ and $A_{\mathrm{MTJ}}$ are the MTJ's tunneling oxide thickness and shape area.

The previous steps of the write operation channel model aredisplayed in Fig.3 and will be further detailed in the rest of this subsection.

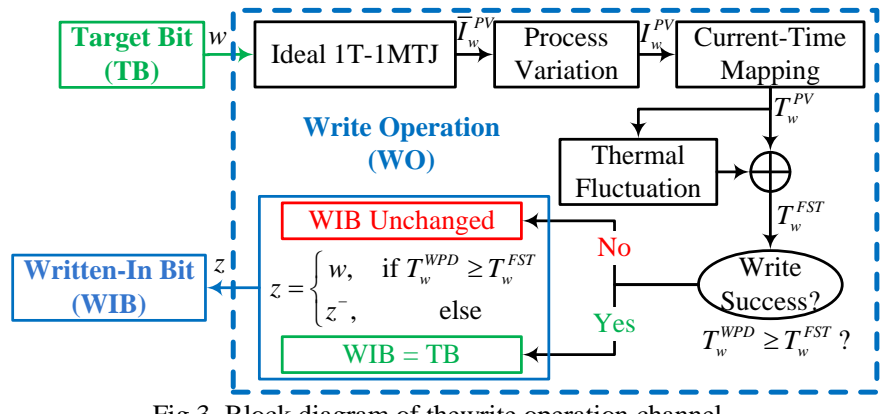

Fig.3. Block diagram of thewrite operation channel.

Thewriting switching current $I_{w}^{P V}$ impacted by the transistor and MTJ process variations can be modeled as a dual-exponential distribution[5]:

$$
p_{P V, w}\left(I_{w}^{P V}\right)=\sigma_{w}^{P V} \exp \left(-\frac{\left|I_{w}^{P V}-\bar{I}_{w}^{P V}\right|}{\sigma_{w}^{P V}}\right),
$$

where $w=0$ (resp. 1)isthe write switching current directionin the $\mathrm{P}\left(\right.$ resp. AP)direction; $\bar{I}_{w}{ }^{P V}$ and $\sigma_{w}^{P V}$ are respectively the mean nominal switching current value and the standard deviation of the corresponding switching currentlisted in TABLE.I.

TABLE.I 1T-1MTJ WRITE CURRENT DISTRIBUTION UNDER TRANSISTOR AND MTJ PROCESS VARIATIONS [25]

\begin{tabular}{|c|c|c|c|c|}
\hline $\begin{array}{c}\text { Transistor } \\
\text { Width }(\mathrm{nm})\end{array}$ & $\bar{I}_{1}^{P V}(\mu \mathrm{A})$ & $\sigma_{1}^{P V}(\mu \mathrm{A})$ & $\bar{I}_{0}^{P V}(\mu \mathrm{A})$ & $\sigma_{0}^{P V}(\mu \mathrm{A})$ \\
\hline 180 & 148.28 & 14.35 & 186.00 & 14.02 \\
\hline 270 & 194.75 & 18.11 & 263.03 & 15.64 \\
\hline 360 & 230.18 & 20.68 & 323.27 & 15.34 \\
\hline 450 & 258.18 & 22.76 & 362.77 & 17.15 \\
\hline
\end{tabular}




\begin{tabular}{|l|l|l|l|l|}
\hline 540 & 280.79 & 24.51 & 387.48 & 19.82 \\
\hline 630 & 299.91 & 26.15 & 404.43 & 21.96 \\
\hline 720 & 315.41 & 27.31 & 416.69 & 23.49 \\
\hline
\end{tabular}

The mapping of step 3) froma mean of the MTJ switching current $I_{w}^{P V}$ toa switching frequency $f_{w}^{P V}$ (reciprocal of the switching time $T_{w}^{P V}$ )for both transitions $0 \rightarrow 1$ and $1 \rightarrow 0$ isgiven inFig.4[25].

The ratio $\sigma_{w}^{T F} / T_{w}^{P V}$ between the standard deviation (STD) $\sigma_{w}^{T F}$ and the mean of the MTJ switching time $T_{w}^{P V}$ versus switching frequency $f_{w}^{P V}$ is shown in Fig.5 for both transitions $0 \rightarrow 1$ and $1 \rightarrow 0$ [25].

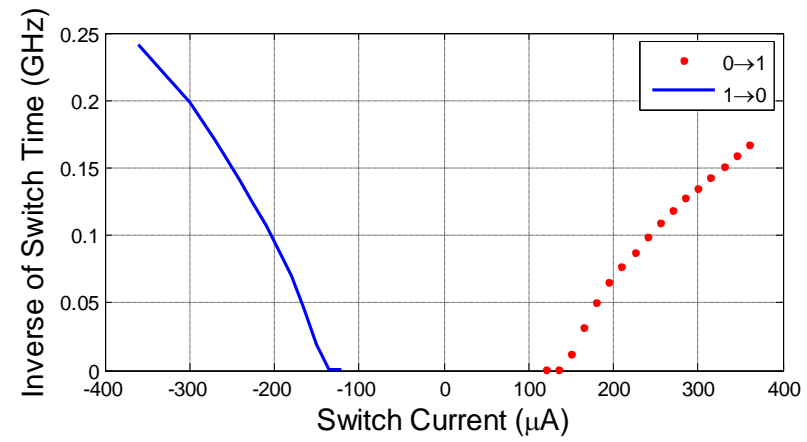

Fig.4. Switching current to switching frequency mapping.

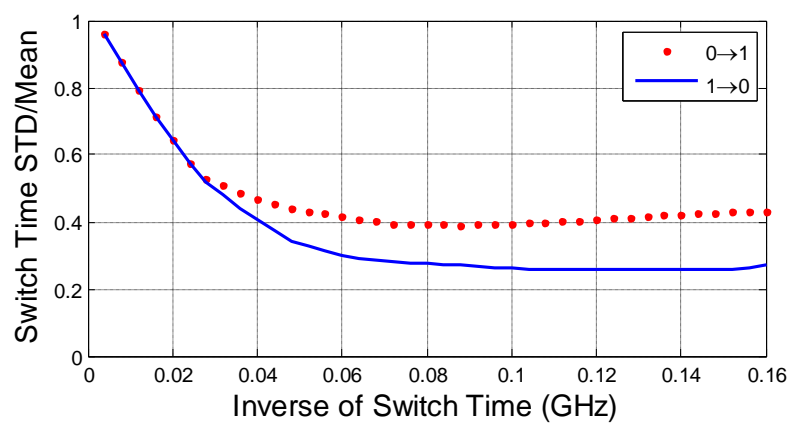

Fig.5. Inverse of switching time to switching time STD/Mean mapping.

As recalled in Section II.D, there are different kinds of thermal fluctuations according to the current switching time at step 4. For a long mean switching time $T_{w}^{P V} \geq 10 \mathrm{~ns}$, thethermal-induced MTJ switching time $T_{w}^{F S T}$ follows approximately theexponential distribution[30], for which the STD and the mean are theoretically always equal. However, for $T_{w}^{P V} \geq 10 \mathrm{~ns}$, it can be observed from Fig.5 that $\sigma_{w}^{T F} / T_{w}^{P V}<1$ (see below $0.1 \mathrm{GHz}$ ). In order to solve this theoretical problem, the random thermal-induced deviation $\Delta T_{w}^{T F}$ is calibrated as:

$$
\Delta T_{w}^{T F}=\sigma_{w}^{T F}\left(\delta_{\mathrm{E}}-1\right),
$$

wherethe probability density function (PDF) of $\delta_{\mathrm{E}}$ is given by:

$$
p_{\text {Exponential }}\left(\delta_{\mathrm{E}}\right)=\exp \left(-\delta_{\mathrm{E}}\right) \text {. }
$$

With this calibration, both the mean and STD of $T_{w}^{F S T}$ satisfy with Fig.4 and Fig.5.

For a very short switching time $T_{w}^{P V} \leq 3 \mathrm{~ns}$, the thermal-induced switching time variation $T_{w}^{F S T}$ followsthe Gaussian distribution[30]:

$$
\Delta T_{w}^{T F}=\sigma_{w}^{T F} \delta_{G},
$$

where :

$$
p_{\text {Gaussian }}\left(\delta_{\mathrm{G}}\right)=\frac{1}{\sqrt{2 \pi}} \exp \left(-\frac{\left(\delta_{\mathrm{G}}\right)^{2}}{2}\right) .
$$

For an intermediate switch time $3 \mathrm{~ns}^{\mathrm{n}}<T_{w}^{P V}<10 \mathrm{~ns}$, the thermal-induced switching time $T_{w}^{F S W}$ is a mixture of the two previous distributions[30]:

$$
\Delta T_{w}^{T F}=\frac{\sigma_{w}^{T F}}{\sqrt{7}}\left(\sqrt{10-T_{w}^{P V}} \delta_{G}+\sqrt{T_{w}^{P V}-3}\left(\delta_{\mathrm{E}}-T_{w}^{P V}\right)\right) .
$$

Therefore, the final switching time $T_{w}^{\text {FSW }}$ follows the distribution characterized by the process variation induced by both the switching time $T_{w}^{P V}$ and the STD.

The write operation failure rate of the STT-MRAM cell at step 5 can be defined as the probability that the write access to the STT-MRAM cell cannot becompleted within a givenwrite pulse duration (WPD) $T_{w}^{W P D}$, i.e., the probability that the given WPD $T_{w}^{W P D}$ is shorter than the final switching time $T_{w}^{F S T}$.

Both, the MTJ's tunneling oxide thickness $t_{\mathrm{ox}}$ and the shape area $A_{\mathrm{MT}}$ follow Gaussian distributions[25]:

$$
\begin{gathered}
p\left(t_{\mathrm{ox}}\right)=\frac{1}{\sqrt{2 \pi} \sigma_{t o x}} \exp \left(-\frac{\left(t_{\mathrm{ox}}-u_{t o x}\right)^{2}}{2\left(\sigma_{t o x}\right)^{2}}\right), \\
p\left(A_{\mathrm{MTJ}}\right)=\frac{1}{\sqrt{2 \pi} \sigma_{\mathrm{AMTJ}}} \exp \left(-\frac{\left(A_{\mathrm{MTJ}}-u_{\mathrm{AMTJ}}\right)^{2}}{2\left(\sigma_{\mathrm{AMTJ}}\right)^{2}}\right),
\end{gathered}
$$

where $u_{t o x}$ and $\sigma_{\text {tox }}$ are the mean and STD of the tunneling oxide thickness, while $u_{u_{\triangle M T \jmath}}$ and $\sigma_{\text {АM } \mathrm{T}}$ are the mean and STD of the shape area.

Taking into account(13), the equivalent resistance of the MTJ with technical variations can be approximatedas:

$$
\begin{aligned}
R_{Z}^{P V} & =\frac{\exp \left(t_{\mathrm{ox}}\right) / A_{\mathrm{MTJ}}}{\exp \left(u_{t o x}\right) / u_{\mathrm{AMTJ}}} R_{Z} \\
& =\left(\frac{u_{\mathrm{AMTJ}}}{A_{\mathrm{MTJ}}}\right) \exp \left(t_{\mathrm{ox}}-u_{t o x}\right) R_{Z} .
\end{aligned}
$$

The technical parameters $\sigma_{t o x}$ and $\sigma_{\text {АM T }}$ in (20)-(22)are obtained from[22]. The other parameters $u_{\text {tox }}$ and $u_{\text {AMTJ }}$ are taken from [25]in which an elliptical shaped $45 \mathrm{~nm} \times 90 \mathrm{~nm}$ in-plane MTJ under a Predictive Technology Model (PTM) $45 \mathrm{~nm}$ model [31]was proposed. These parameters were calibrated with the measurement data from a leading magnetic recording company and are recalled in TABLE.II.

\begin{tabular}{|c|c|c|c|}
\hline \multicolumn{4}{|c|}{ TABLE.IIMTJ and Transistor Technical Parameters } \\
\hline $\begin{array}{c}\text { Device } \\
\text {-stor }\end{array}$ & Parameter & Mean & STD \\
\cline { 2 - 4 } & Channel Length $L_{T}(\mathrm{~nm})$ & 45 & \\
\cline { 2 - 4 } & Channel Width $W_{T}(\mathrm{~nm})$ & 180 to 720 & \\
\hline \multirow{4}{*}{ MTJ } & Threshold voltage $V_{t h}(\mathrm{~V})$ & 0.466 & \\
\cline { 2 - 4 } & Shape Area $A_{\mathrm{MTJ}}\left(\mathrm{nm}^{2}\right)$ & $45 \times 90 \pi / 4$ & $5 \%$ of mean \\
\cline { 2 - 4 } & MgO Thickness $t_{\mathrm{ox}}(\mathrm{nm})$ & 1.5 & $2 \%$ of mean \\
\cline { 2 - 4 } & Low Resistance $R_{\mathrm{P}}(\mathrm{Ohm})$ & 1000 & \\
\cline { 2 - 4 } & High Resistance $R_{\mathrm{AP}}(\mathrm{Ohm})$ & 2000 & \\
\cline { 2 - 4 } & $\begin{array}{c}\text { Reference Resistance } R_{\mathrm{REF}} \\
(\mathrm{Ohm})\end{array}$ & 1500 & \\
\hline
\end{tabular}


The STD $\sigma_{v_{t h}}$ of the threshold voltage $v_{t h}$ (seeTABLE.II)is approximately computed as[25]:

$$
\sigma_{v_{\text {th }}}=0.3 \frac{45^{2}}{W_{T} L_{T}}=\frac{607.5}{W_{T} L_{T}},
$$

where $W_{T}$ and $L_{T}$ are respectively the transistor width and length in $\mathrm{nm}$.

Let us turn now to the evaluation of the write channel capacity. Given the equiprobableassumption made over the input TB $w$, the capacity of the write channel can be written as:

$$
\begin{aligned}
I\left(w ; R_{z}\right) & =H\left(R_{z}\right)-H\left(R_{z} \mid w\right) \\
& =-\int p\left(R_{z}\right) \log _{2}\left(p\left(R_{z}\right)\right) d R_{z} \\
& +\sum_{w=0}^{1} \int p\left(R_{z} \mid \mathrm{w}\right) p(w) \log _{2}\left(p\left(R_{z} \mid \mathrm{w}\right)\right) d R_{z},
\end{aligned}
$$

wherethe key terms $p\left(R_{z}\right)$ and $p\left(R_{z} \mid w\right)$ are given by:

$$
\begin{gathered}
p\left(R_{z}\right)=\sum_{w=0}^{1} p(w) p\left(R_{z} \mid w\right), \\
p\left(R_{z} \mid w\right)=\sum_{z^{-}=0}^{1} p\left(z^{-}\right) p\left(R_{z} \mid w, z^{-}\right) ;
\end{gathered}
$$

$p(z)$, according to the previous state distribution $p\left(z^{-}\right)$can be computed as:

$$
p(z)=\sum_{w=0}^{1} p(w) \sum_{z^{-}=0}^{1} p\left(z^{-}\right) p\left(z \mid w, z^{-}\right) ;
$$

From Section II.A and step 5of Section III.A, the transition probability $p\left(z \mid w, z^{-}\right)$can be expressed in terms of write success and fail probabilities, i.e. :

$$
\left\{\begin{array}{c}
p\left(z=0 \mid w=0, z^{-}=0\right)=p\left(z=1 \mid w=1, z^{-}=1\right)=1 \\
p\left(z=1 \mid w=0, z^{-}=0\right)=p\left(z=0 \mid w=1, z^{-}=1\right)=0 \\
p\left(z=0 \mid w=0, z^{-}=1\right)=p(\mathrm{P} \text { w rite success }) \\
p\left(z=1 \mid w=1, z^{-}=0\right)=p(\text { A P w rite success }) \\
p\left(z=1 \mid w=0, z^{-}=1\right)=p(\mathrm{P} \text { write fail }) \\
p\left(z=0 \mid w=1, z^{-}=0\right)=p(\text { AP write fail })
\end{array}\right.
$$

Substituting (28)into (27), $p(z=0)$ and $p(z=1)$ can be further written as:

$$
\begin{aligned}
p(z=0) & =p(w=0) p\left(z^{-}=0\right) \\
& +p(w=0) p\left(z^{-}=1\right) p(\mathrm{P} \text { w rite success }) \\
& +p(w=1) p\left(z^{-}=0\right) p(\text { A P w rite fail }), \\
p(z=1)= & p(w=1) p\left(z^{-}=1\right) \\
& +p(w=0) p\left(z^{-}=1\right) p(\mathrm{P} \text { w rite fail }) \\
& +p(w=1) p\left(z^{-}=0\right) p(\text { A P write success })
\end{aligned}
$$

Since the previous state $z^{-}$has asymptotically the same distribution as $z,(29)$ can be reformulated as:

$$
\begin{aligned}
& p(w=0) p\left(z^{-}=1\right) p(\mathrm{P} \text { write success }) \\
& =p(z=0)-p(w=0) p\left(z^{-}=0\right)- \\
& \quad p(w=1) p\left(z^{-}=0\right) p(\text { AP w rite fail }) \\
& =p(w=1) p\left(z^{-}=0\right)-p(w=1) p\left(z^{-}=0\right) p(\text { AP w rite fail })
\end{aligned}
$$

$$
\begin{aligned}
& =p(w=1) p\left(z^{-}=0\right)[1-p(\text { A P w rite fail })] \\
& =p(w=1) p\left(z^{-}=0\right) p(\text { A P write success }) .
\end{aligned}
$$

Since $p(w=0)=p(w=1)=0.5$, the ratio of $p\left(z^{-}=1\right)$ and $p\left(z^{-}=0\right)$ can readily be obtained as:

$$
\frac{p\left(z^{-}=1\right)}{p\left(z^{-}=0\right)}=\frac{p(\text { A } \mathrm{P} \text { write success })}{p(\mathrm{P} \text { write success })} .
$$

(32) simplymeans thatthe WIB $z$ distribution depends only on the write operation success rate and that the state distribution ratio is exactly equal to the ratio of the anti-parallel and parallelwrite success probabilities.In other words, with no a priori information onthe $\mathrm{TB}$, the distribution of the WIB converges to the distribution given by(32). Therefore, theequiprobable assumption does not hold anymore for theWIB $z$.

Thuscomputing the capacity(24)involvesto obtain $p$ (write success) and thePDF $p\left(R_{z} \mid w, z^{-}\right)$, which can be achieved by Monte-Carlo simulations of the proposed write channel model.

\section{B. Read Channel Model}

Due to the unbalanced driving ability of the transistor,the failure probability of anti-parallel $(0 \rightarrow 1)$ writing is much higher than that of parallel $(1 \rightarrow 0)$ writing. However, the higher write operation failure probability gives a favor of lower flipping probability to the read operation. Therefore, differently from the write channel, the AP direction is preferable to the $\mathrm{P}$ directionfor read operation.

The read operation channel model can also be divided into 5 consecutive steps:

1) Generate the mean read current value $\bar{I}_{A P}^{P V}$;

2) Add a random variation to the mean read current $\bar{I}_{A P}^{P V}$ so as to generate the process variation affected read current $I_{A P}^{P V}$;

3) Map the process variation affected read current $I_{A P}^{P V}$ to a flipping time $T_{A P}^{P V}$;

4) Generate the overallswitching time $T_{A P}^{F S T}$ by further introducing a random thermal-induced deviation $\Delta T_{A P}^{T F}$ to the previous switching time $T_{A P}^{P V}$ :

$$
T_{A P}^{F S T}=T_{A P}^{P V}+\triangle T_{A P}^{T F}
$$

5) Finally, a readflipping error is decided by comparing the given read pulse duration (RPD) $T_{A P}^{R P D}$ with the required flipping time $T_{A P}^{F S T}$. If $T_{A P}^{R P D} \geq T_{A P}^{F S T}$, the written-in bit $z$ is flipped to 1 and the read-out bit state $r=1$; otherwise, no flipping error occurs and the $\mathrm{ROB}_{r}$ is updated as the WIB $z$ :

$$
r=\left\{\begin{array}{cc}
1, & \text { if } T_{A P}^{R P D} \geq T_{A P}^{F S T} \\
z, & \text { else }
\end{array} .\right.
$$

Moreover, the decidedbit (DB) $\hat{r}$ is obtained by comparing the actual sensing current $I(\hat{r})$ with an ideal sense current $I_{\text {REF }}$ : 


$$
\hat{r}=\left\{\begin{array}{cc}
0 & \text { if } I(\hat{r})>I_{\mathrm{REF}} \\
1 & \text { else }
\end{array},\right.
$$

where $I(\hat{r})$ and $I_{\mathrm{REF}}$ are:

$$
\begin{aligned}
& I_{\hat{r}}=\frac{V_{\text {sense }}-V_{t h}}{R_{r}}, \\
& I_{\text {REF }}=\frac{V_{\text {sense }}-\bar{V}_{t h}}{R_{\text {REF }}} ;
\end{aligned}
$$

$V_{\text {sense }}=0.58 \mathrm{~V}$ is the sense voltage for reading, $\bar{V}_{t h}=$ $0.466 \mathrm{~V}$ (see TABLE.II) is the mean threshold voltage, $V_{t h}$ is the actual threshold voltage depending of process variation, $R_{r}$ is the actual resistance value corresponding to ROB state $r$ and $R_{\mathrm{REF}}=1500 \mathrm{Ohm}$ (see TABLE.II).Due to the $V_{t h}$ variation, the nominal resistance value for the bit decision is:

$$
R_{\dot{r}}=\frac{V_{\text {sense }}-0.466}{V_{\text {sense }}-V_{t h}} R_{r} .
$$

It should be mentioned that there are many kinds of sense amplifiers [32]-[35] and none of them has really become a "standard" cell. Because of this, the sense amplifier in the read channel is assumed to be an ideal current sense amplifier with a reference current value simply being the mean of the current values of the low and high resistance states; in other words, this sense amplifier does neither take into account the process variations, nor the thermal fluctuations.

Apart from thecurrent direction and the current strength, the read operation is analogous to the write operation (see Fig.6). In this way, most of the technical parameters and all the distribution models already used for the write channel can be used again for the read channel.and the reference resistance is assumed to be ideal with neither process variations nor thermal fluctuations.

For the AP read operation over the $1 \mathrm{~T}-1 \mathrm{M}$ cell, there are three types of reading errors:

1) The cell stores a 0 but is read out as a 1 ;

2) The cell stores a 1 but is read out as a 0 ;

3) The cell stores a 0 but is flipped to 1 .

The capacity of the read channel can be written as:

$$
\begin{aligned}
I\left(z ; R_{\hat{r}}\right) & =H\left(R_{\hat{r}}\right)-H\left(R_{\hat{r}} \mid z\right) \\
& =-\int p\left(R_{\hat{r}}\right) \log _{2}\left(p\left(R_{\hat{r}}\right)\right) d R_{\hat{r}} \\
& +\sum_{z=0}^{1} \int p\left(R_{\hat{r}} \mid z\right) p(z) \log _{2}\left(p\left(R_{\hat{r}} \mid z\right)\right) d R_{\hat{r}},
\end{aligned}
$$

where the resistance distribution $p\left(R_{\hat{r}}\right)$ of the nominal resistance value in (38) can be written as:

$$
p\left(R_{\hat{r}}\right)=\sum_{z=0}^{1} p(z) p\left(R_{\hat{r}} \mid z\right),
$$

and $p(z)$ is obtained from (32) by computing $p$ (w rite success). Therefore, in order to evaluate the $\operatorname{PDF} p\left(R_{\hat{r}} \mid z\right)$ and to compute (39), one has to simulate both the write and read operations.

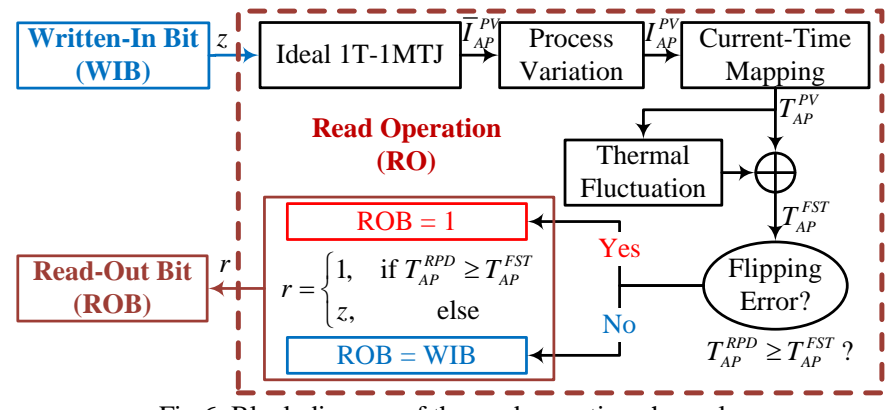

Fig.6. Block diagram of the read operation channel.

\section{Write and Read Channel Capacity}

The combinedwrite and read channel capacity can be written as:

$$
\begin{aligned}
I\left(w ; R_{\hat{r}}\right) & =H\left(R_{\hat{r}}\right)-H\left(R_{\hat{r}} \mid w\right) \\
& =-\int p\left(R_{\hat{r}}\right) \log _{2}\left(p\left(R_{\hat{r}}\right)\right) d R_{\hat{r}} \\
& +\sum_{w=0}^{1} \int p\left(R_{\hat{r}} \mid w\right) p(w) \log _{2}\left(p\left(R_{\hat{r}} \mid w\right)\right) d R_{\hat{r}},
\end{aligned}
$$

where $p\left(R_{\hat{r}}\right)$ and $p\left(R_{\hat{r}} \mid w\right)$ are:

$$
\begin{aligned}
p\left(R_{\hat{r}}\right) & =\sum_{w=0}^{1} p(w) p\left(R_{\dot{r}} \mid w\right), \\
p\left(R_{\hat{r}} \mid w\right) & =\sum_{z^{-}=0}^{1} p\left(z^{-}\right) p\left(R_{\hat{r}} \mid w, z^{-}\right) \\
& =\sum_{z^{-}=0}^{1} p\left(z^{-}\right) \sum_{z=0}^{1} p\left(R_{\hat{r}}, z \mid w, z^{-}\right) \\
& =\sum_{z^{-}=0}^{1} p\left(z^{-}\right) \sum_{z=0}^{1} p\left(z \mid w, z^{-}\right) p\left(R_{\hat{r}} \mid z\right) .
\end{aligned}
$$

Similarly to SectionIII.A andSection III. B, $p\left(z \mid w, z^{-}\right)$and $p\left(R_{\hat{r}} \mid z\right)$ are computed by simulating the write channel and the read channel respectively.

\section{SimUlations AND RESUlts}

In this section, the proposed channel model is first validated by comparing simulation results to the experimental results published in[25]. After validation, the reliability of the 1T-1MTJ operation channel is evaluated in terms of probability density function, operation failure rate, bit error rate and channel capacity.All the process variations and thermal fluctuations mentioned in SectionIII are included in these simulations.

\section{A. Model Validation}

In this subsection, all the curves with "REF" represent the original experimental results published in [25], whereas the curves with "SIMU" represent the recreated results via the proposed channel model.

Fig.7 and Fig.8 (see Fig. 7 (a) in [25]) show our simulation results of the write error rates (WERs) with awriting pulse duration (WPD) equal to $10 \mathrm{~ns}$ and $20 \mathrm{~ns}$. It can be observed that the recreated results obtained by the proposed STT-MRAM operation model follow closely the already published corresponding results. 


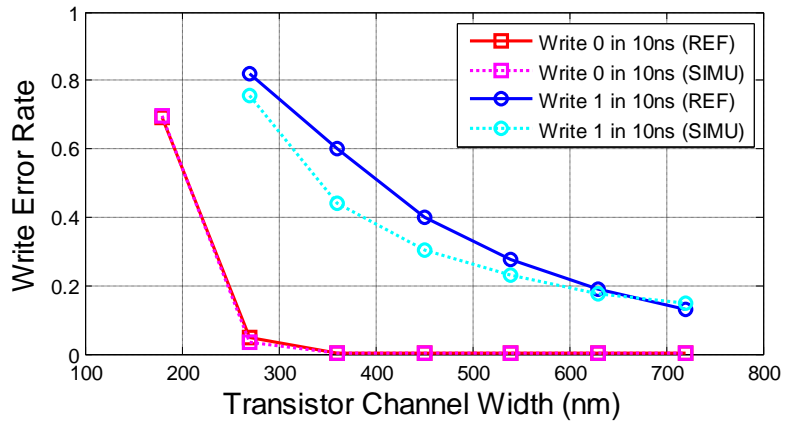

Fig.7. Write error rate for a $10 \mathrm{~ns}$ writing pulse width.

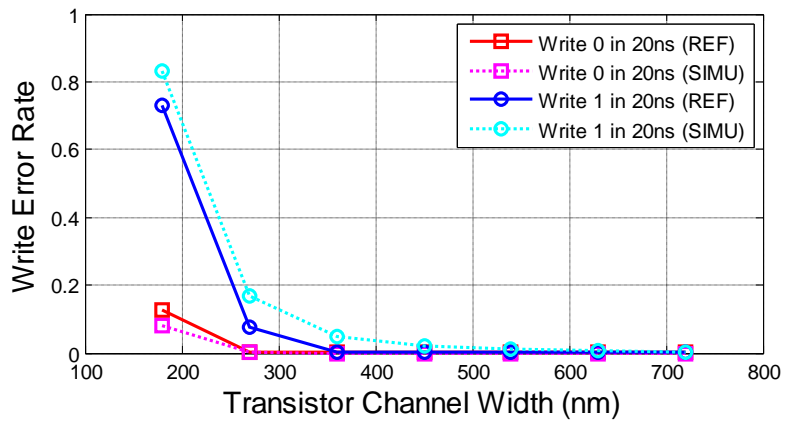

Fig.8. Write error rate for a $20 \mathrm{~ns}$ writing pulse width.

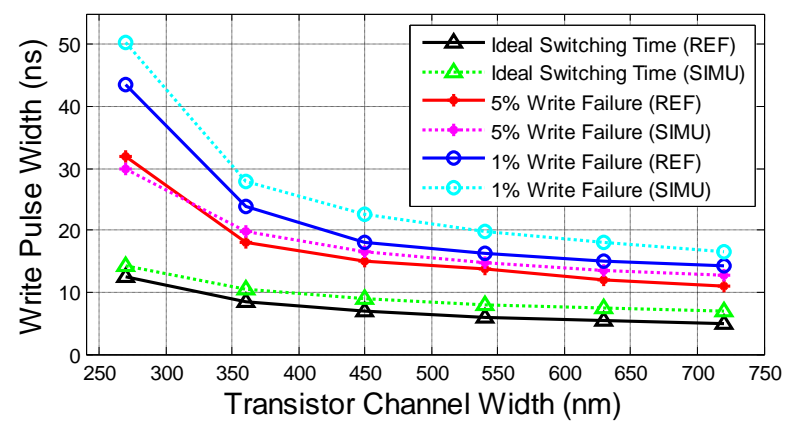

Fig.9. Comparison of writing "1" error rates between our model and Fig. 7 (b) of [25].

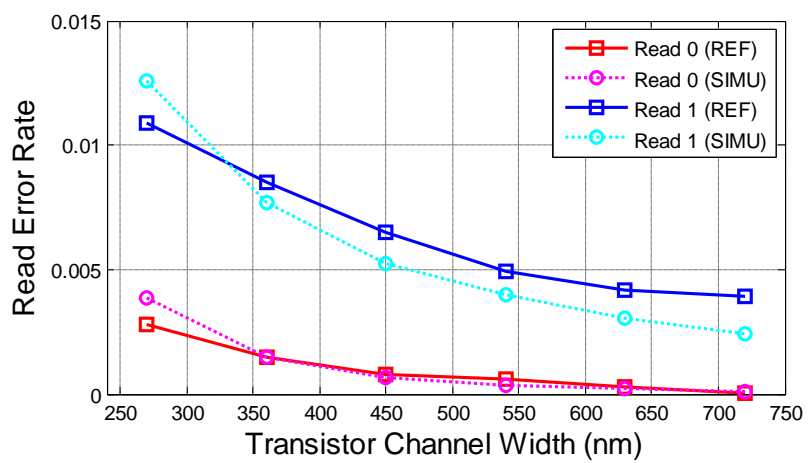

Fig.10. Reading failure rate for different transistor widths.

Fig.9 (see Fig. 7 (b) in [25]) displays the required WPDs for different NMOS transistor widths. In this figure, the ideal switching time represents the results based on the mean device parameters without considering any process variations and thermal fluctuations. It can be observed that the recreated $1 \%$ and 5\% WER switching time also follow closely the corresponding already published curves. The limited differences between the $1 \%$ (resp. 5\%) WER switching time curves are mainly due to the small difference between the recreated ideal switching time and the corresponding published ideal switching time.

Fig.10 (see Fig. 3 in [25]) gives the read error rates (RERs) for different transistor widths. As [25] uses a practical sense amplifier, the recreated results with an ideal sense amplifier lead to slightly better results in most cases.

\section{B. Write Operation Channel}

In this subsection, the write channel is evaluated according tothe model illustrated by Fig.3in SectionIII.A.The TBs are assumed to be equiprobable. We recall that the switching current parameters for the write operations are listed in TABLE.I, the conversion from switching current to switching time is displayed inFig.4 and the thermal-induced switching timeis generated by using (15)-(19).

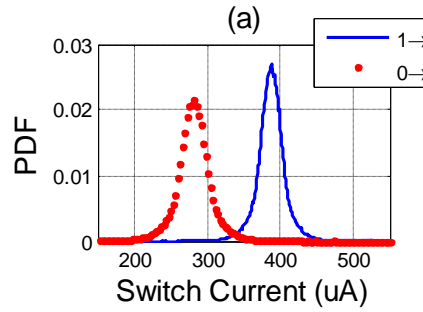

(c)
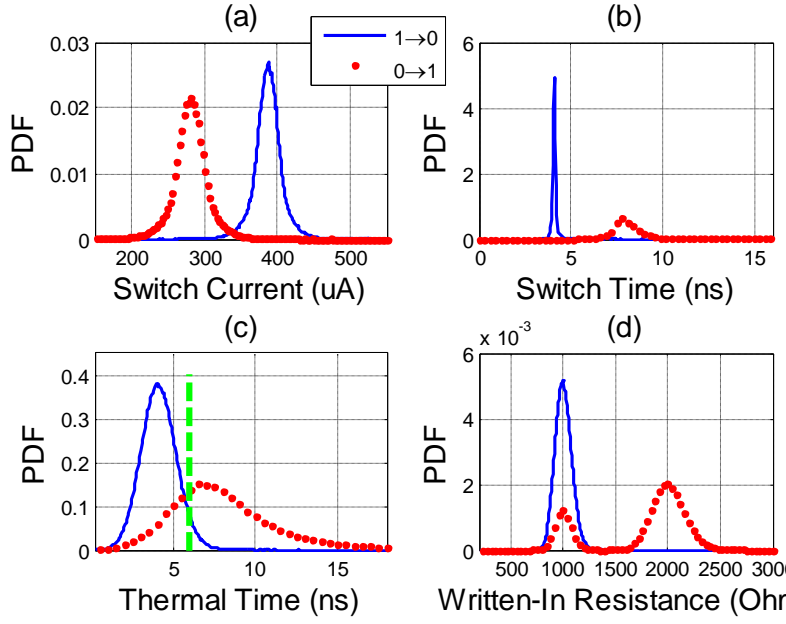

(d)

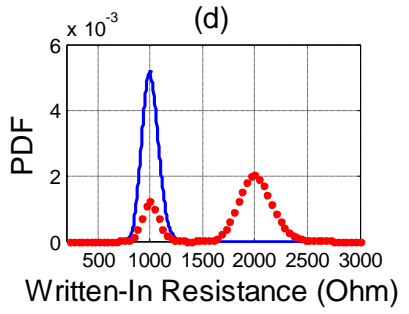

Fig.11. STT-MRAM write operational channel (transistor size $=540 \mathrm{~nm}$, WPD $=10 \mathrm{~ns}$ ).

(a)

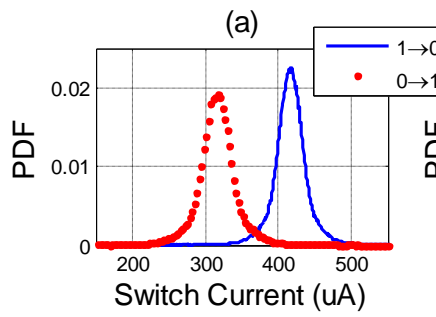

(c)
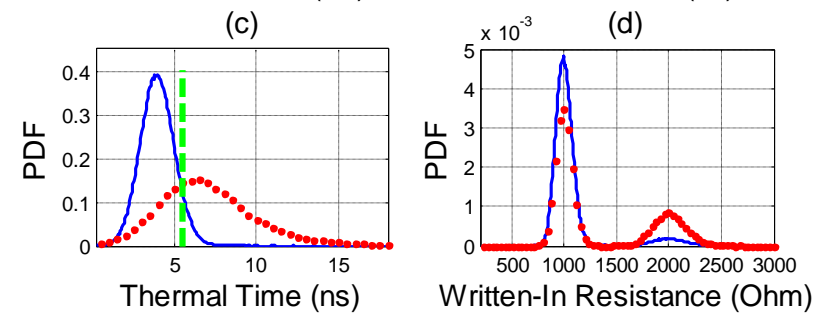

(b)

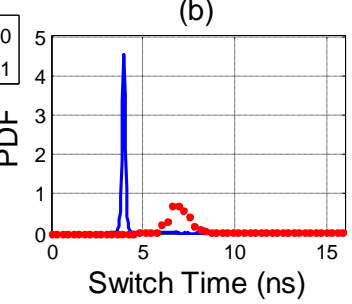

Fig.12. STT-MRAM write operational channel (transistor size $=720 \mathrm{~nm}$, WPD $=5.5 \mathrm{~ns}$ ).

Fig.11 displays the whole process of the write operation channel with a $540 \mathrm{~nm}$ transistor size; Fig.11 (a) presents the distributions of write currents $I_{w}^{P V}$ under the impact of process variations, (see (14)). Fig.11 (b) illustrates the distribution of switch time $T_{w}^{P V}$ mapped from switching current $I_{w}^{P V}$ (see Fig.4 and step 3 in Section III.A), where the larger deviation for the AP direction $(0 \rightarrow 1)$ can be easily observed. Fig.11 (c) 
highlights even larger expansions of the final switching time $T_{w}^{F S T}$ of (11) affected by thermal fluctuations; a large difference can be noticed between Fig.11 (b) and Fig.11 (c) with and without thermal fluctuations, respectively. Moreover, in Fig.11 (c), a green dash line indicates a given WPD $=10 \mathrm{~ns}$ serving as the boundary between a write operation success (left-hand side) region with required cell flipping time inferior to this given WPD and a write operation failure (right-hand side) region. Fig.11 (d) gives the written-in resistance distributions generated from (22) for a WPD $=10 \mathrm{~ns}$. The write failure for the $0 \rightarrow 1$ transition, i.e., the small red peak around the low resistance state (around $1000 \mathrm{Ohm}$ ) can be clearly observed, and this peak stems from the large tail existing at the right-hand side of the green $10 \mathrm{~ns}$ dash line in Fig.11 (c) for too long switching durations. Therefore, for WPD=10 ns, the high write operation failure rate can be predicted.

Fig.12 display the whole process of the write operation channel with even more critical parameters $(720 \mathrm{~nm}$ transistor size and WPD $=5.5 \mathrm{~ns}$ ). Differently from Fig.11, due to the insufficient WPD, the reliability of writing " 0 " is also affected and a write operation failurefor both transitions can be observed in the lower subfigures (c) and (d).

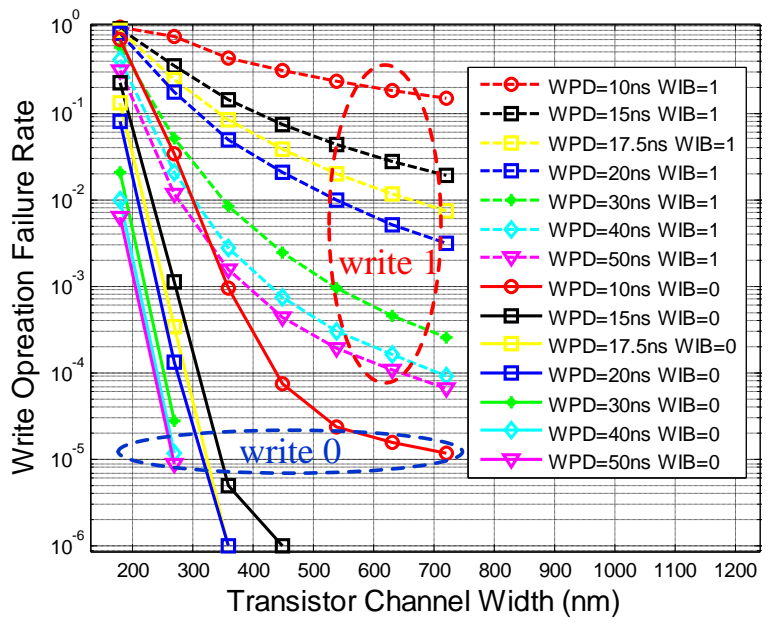

Fig.13. STT-MRAM writeoperation failure rates with different transistor widths and different WPDs.

Fig.13 shows the write operation failure rates for different transistor widths and different WPDs. It is easy to notice that the write failure rate for the $0 \rightarrow 1$ transition is several orders of magnitude higher than the failure rate for the $1 \rightarrow 0$ transition. The larger the transistor, the larger the drive current strength so that the required switching time is shorter and thus the write operation failure rate tends to be lower. Similarly to increasing the transistor width, the same improvement for the write operation failure can be easily observed by increasing the WPD. Since the operation failure rate involves only $0 \rightarrow 1$ and $1 \rightarrow 0$ transitions, the performance is not influenced by the original cell state before writing operation.Differently from Fig.13, Fig.14 measures the written-in bit error rate and the corresponding simulations logically involves the original cell state before the writing operation. Therefore, the fact that the TB can be successfully written into the STT-MRAM cell depends also of the original cell state. To simplify the simulations, we assume that there are originally as much "0"s as "1"s.

Fig.15 displays the write channel capacity (see (24)) for various transistor widths and various WPD. Similarly to Fig.14, since Fig. 15 is related with the bit reliability, the capacity simulations also involve the original cell state. From Fig.15for a $\mathrm{WPD}=10 \mathrm{~ns}$, a target channel code rate equal to 0.85 cannot meet the capacity requirement due to the too high write failure rate of the AP direction; moreover, solutions with transistor widths inferior to $200 \mathrm{~nm}$ can neither satisfy the system requirements. As the target code rate slightly increases to 0.9 , solutions can only be selected among the designs with transistor width being superior to $360 \mathrm{~nm}$.

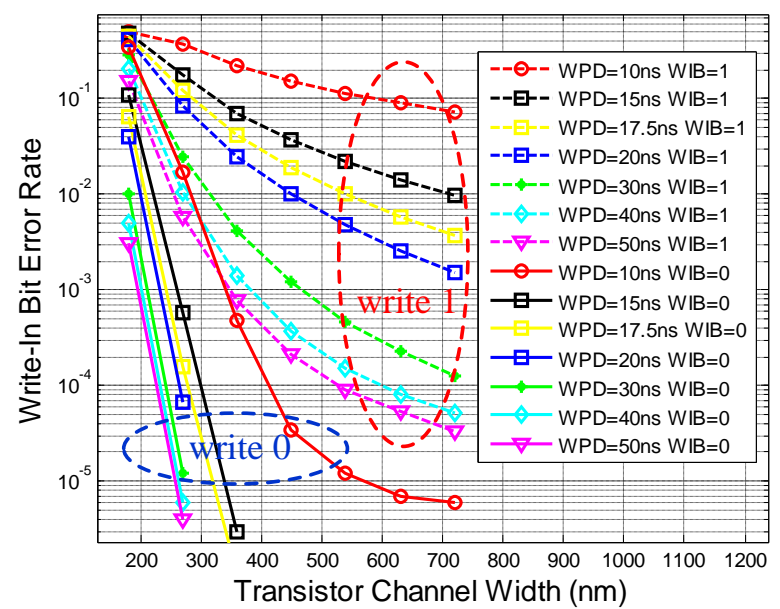

Fig.14. STT-MRAM write-in bit error rates with different transistor widths and different WPDs.

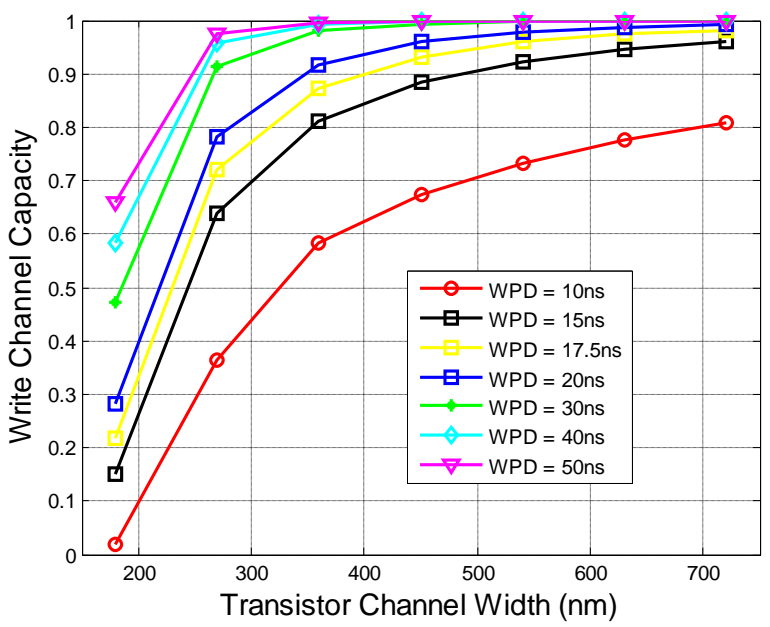

Fig.15. STT-MRAM write channel capacities with different transistor widths and different WPDs

Fig.16 illustrates the result given by(32). It shows that the distribution of the WIB is not equiprobable in general and that writing ' 0 ' is always easier than writing ' 1 '. Moreover, solutions with WPD $=10 \mathrm{~ns}$ and solutions with a transistor width smaller than $270 \mathrm{~nm}$ cause large differences between $p(z=0)$ and $p(z=1)$. By comparing the results ofFig.13 with Fig.16, it can be further observed that the WIB approaches to the equiprobability as the write operation failure rate decreases. It 
can then be concluded by comparing with Fig.15 that the hypothesis that WIBs areequiprobableholds for reliable write channels with write channel capacity being superior to 0.9 $\mathrm{bit} / \mathrm{cell}$.

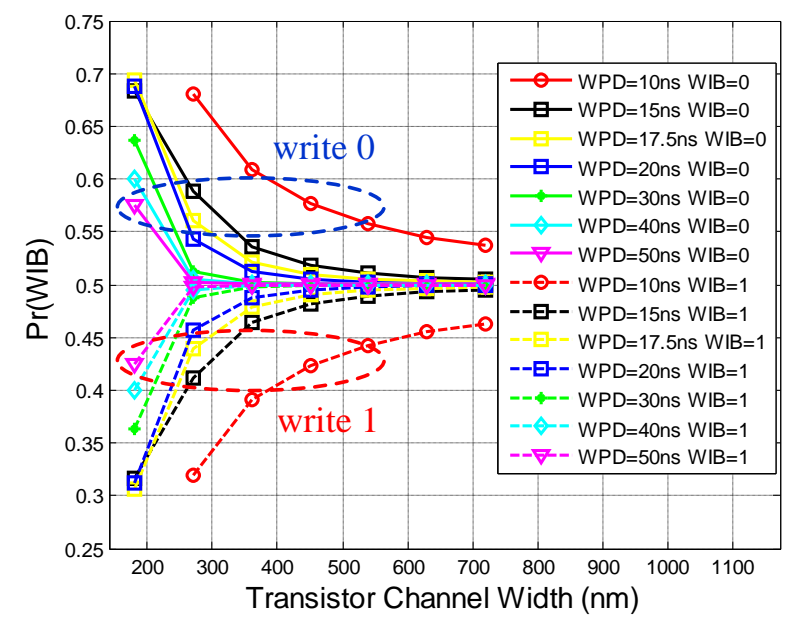

Fig.16. STT-MRAM written-in bits (WIBs) distributions with different transistor widths and different WPDs.

\section{Read Operation Channel}

In this subsection, the read channel is evaluated according to the model illustrated by Fig.6in SectionIII.B. The WIBsare assumed to be uniformly distributed to eliminate any write channel influence. Theparameters for the read operation are listed inTABLE.IIand are applied to(20)-(23).

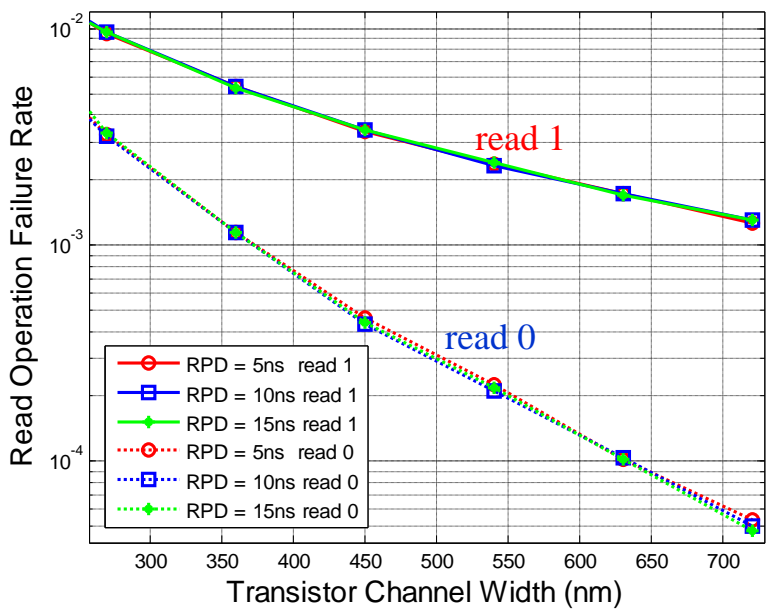

Fig.17. STT-MRAM read operation failure rates with different transistor widths and different RPDs.

Fig.17 and Fig.18 present the read operational failure rate and the read channel capacity for different transistor widths and for different read pulse durations (RPDs). Because of the lowvalues taken by read currents, the flipping error i.e., the third type of read error nearly never happens even with $\mathrm{RPD}=15 \mathrm{~ns}$ and operational failures are mainly due to process variations of the MTJ resistanceand to the threshold voltage variations. As the threshold voltage STD decreaseswhenthe transistor width increases, the operation failure rate for a large transistor width is better than the rate for a small transistor width. Since the MTJ resistance distribution is independent of thetransistor width, the read failure rate is no affected by the short RPDs. Moreover, because of the larger resistance variations of state ' 1 ', thefailure rate to read a ' 1 ' is higher than that to read a ' 0 '.Theread channel capacity ofFig.18 is much higher than the write channel capacity of Fig.15; this is due to the small current values used for read operations, and consequently the flipping error rate is near zero.

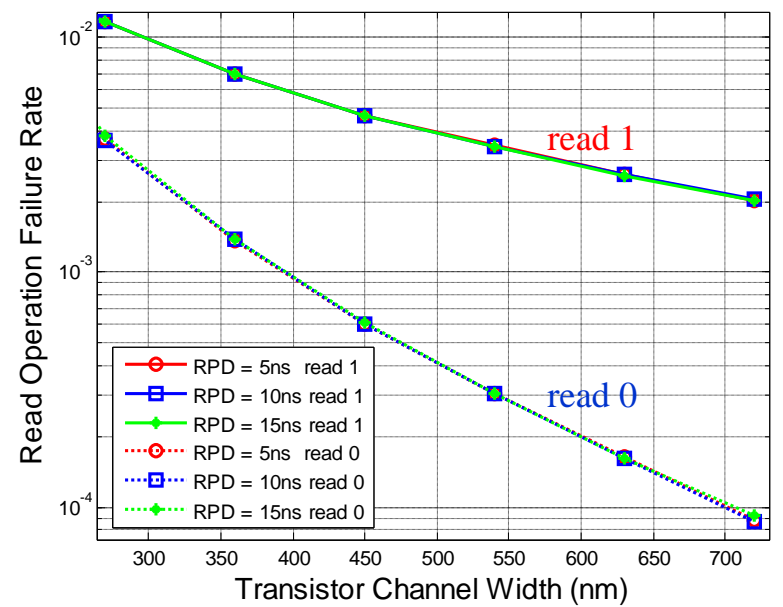

Fig.18. STT-MRAM read channel capacitywith different transistor widths and different RPDs.

\section{Combined Write and Read Channel}

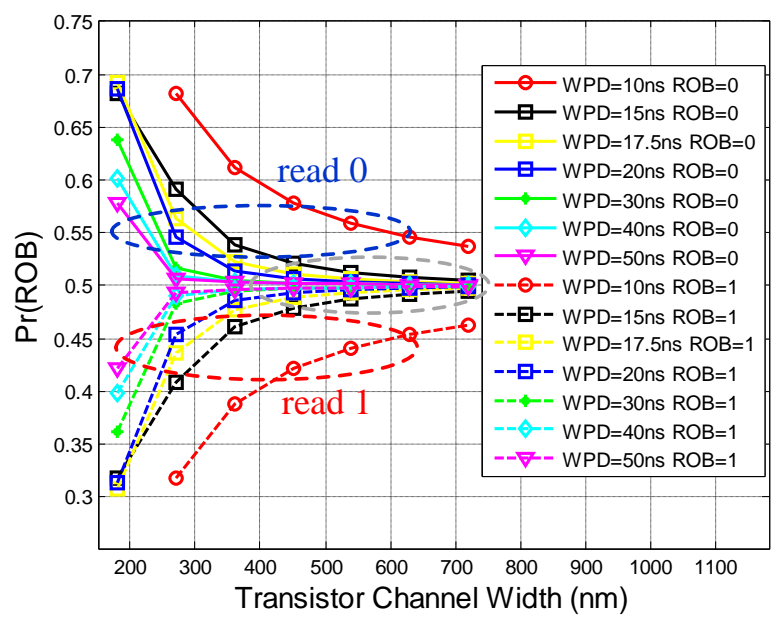

Fig.19. STT-MRAM read-out bits (ROBs) distributions with different transistor widths and different WPDs (RPD $=5 \mathrm{~ns}$ ).

Fig.19 gives the read-out bit (ROB) distributions after that the cell write and read operations are both completed. It can be seen that the ROB distributions are similar to the WIB distributions in Fig.16. However, as the channel width increases, the ROB distribution difference is slightly larger than the WIB distribution difference (compare the curves within the grey dashed circle in Fig.19 with the corresponding curves in Fig.16). This fact comes from the difference of MTJ resistance deviations for state ' 0 ' and state ' 1 ' due to process variations of the MTJ's shape surface and tunnel oxide thickness. However, the equiprobable assumption still approximately holds for the cases of a transistor size larger than $270 \mathrm{~nm}$ and a WPD longer than $10 \mathrm{~ns}$.

Fig.20 and Fig.21respectively present the operation failure rate and the BER of the combined write and read operation channel. Due to the process variations of the MTJ resistance, 
there exists error floorsfor both parallel and anti-parallel directions. Note that the intrinsic resistance variations cannot be removed by changing extrinsic parameters such as the transistor size orthe WPD; thereforeusing an ECC becomesabsolutely compulsorywhen the BER does not meet the target requirement.

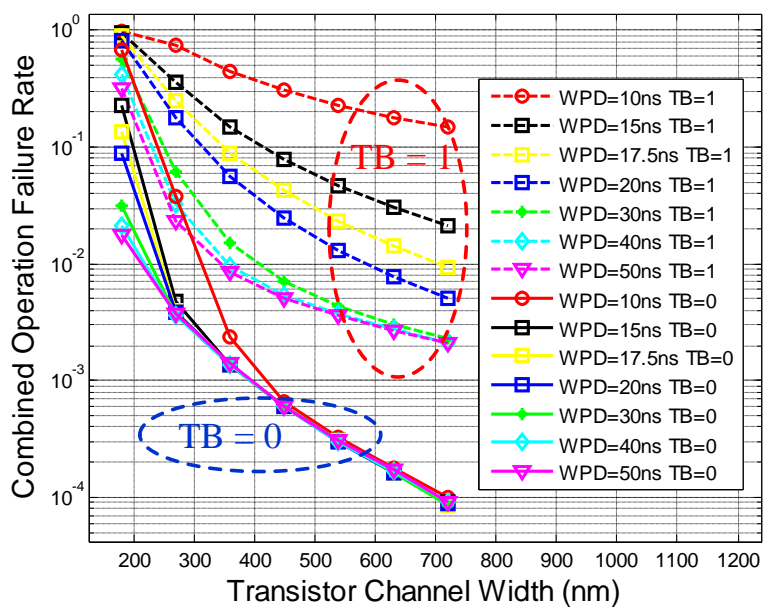

Fig.20. STT-MRAM written-in bits (WIBs) distributions with different transistor widths and different WPDs $(\mathrm{RPD}=5 \mathrm{~ns})$.

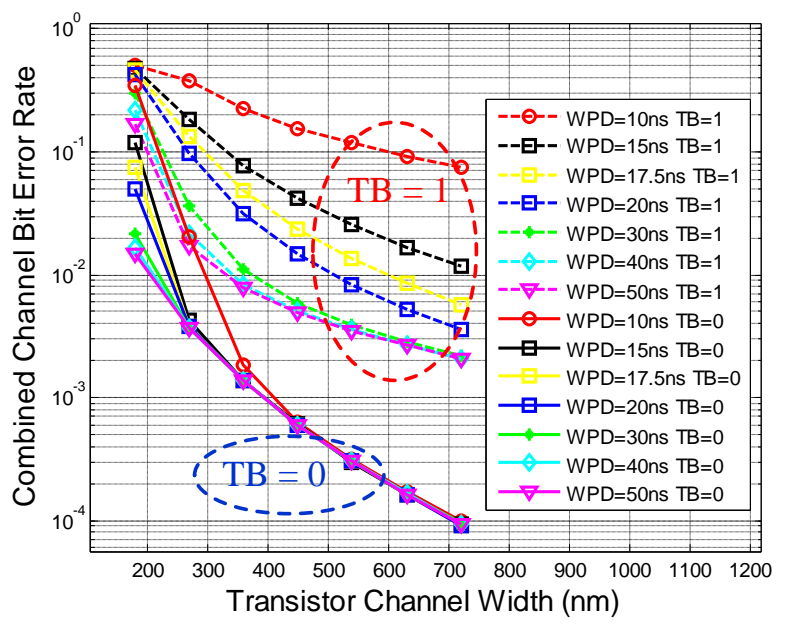

Fig.21. STT-MRAM written-in bits (WIBs) distributions with different transistor widths and different WPDs ( $\mathrm{RPD}=5 \mathrm{~ns}$ ).

Fig.22 gives the combined channel capacities, i.e., the maximum bit number that can be reliably written in and read out in one cell. This metric gives the upper bound for the channel coding rate with infinite code length. Note that as the transistor drive capacity increases, the reduced operation failure rate and the increased channel capacity indicate that an ECC can have less redundancy (i.e., higher efficiency) to protect messages. If the target code rate is 0.7 , the transistor width needs to be larger than $270 \mathrm{~nm}$ and the WPD has to be kept longer than $17.5 \mathrm{~ns}$. If the target code rate is 0.9 , the minimum transistor width is $350 \mathrm{~nm}$ for a minimum WPD equal to $20 \mathrm{~ns}$.

Finally, Fig.23 gives the outage probability for an outage capacity $C_{o}=0.9 \mathrm{bit} /$ cell with different transistor widths, different block lengths (BLK) and different WPDs. The ergodic curves of different WPDs and different transistor sizes serve as limits. The lowest outage probabilities simulated for different WPDs and all transistor sizes reach at least the level of $10^{-7}$. In other words, if a point is not plotted, it simply means that the performance is below $10^{-7}$.

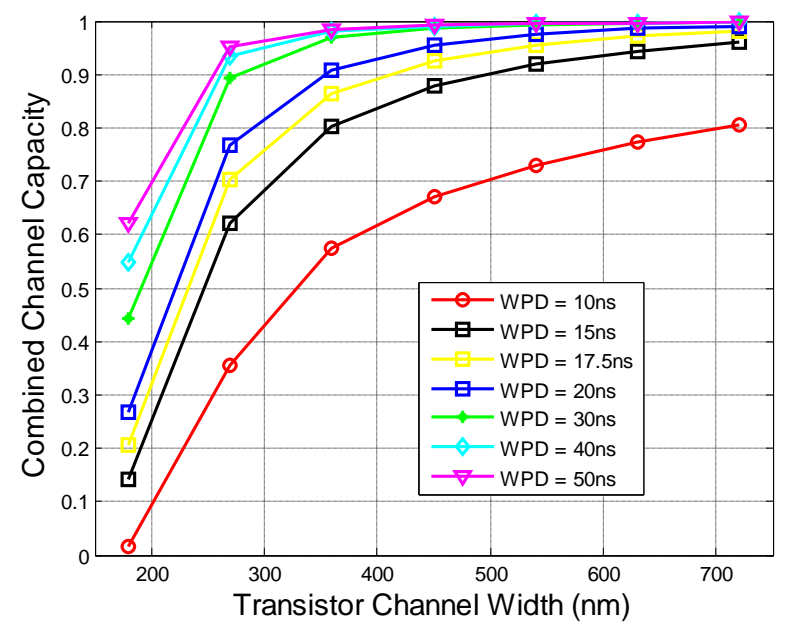

Fig.22. STT-MRAM written-in bits (WIBs) distributions with different transistor widths and different WPDs (RPD $=5 \mathrm{~ns})$.

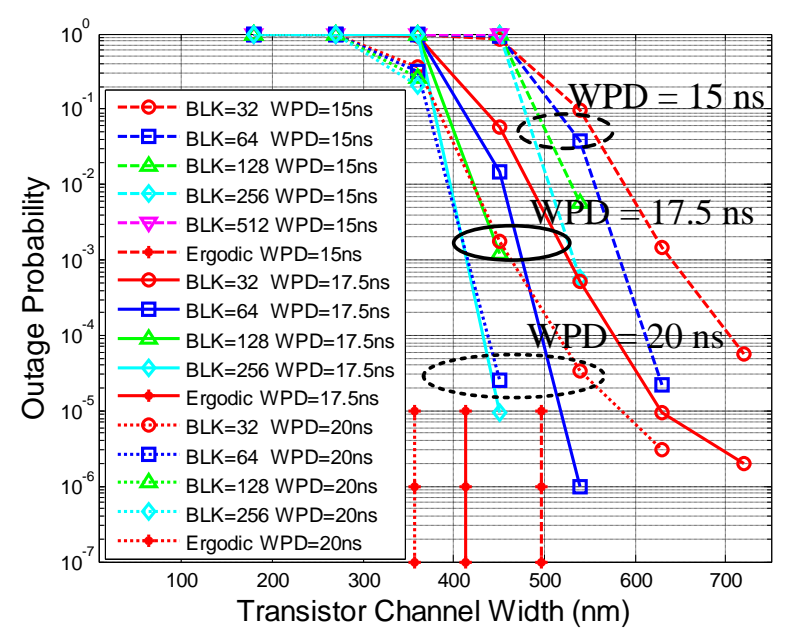

Fig.23. STT-MRAM outage probability as outage capacity $C_{o}=0.9 \mathrm{bit} / \mathrm{cell}$ with different transistor widths (including infinite block length) and different WPDs $(\mathrm{RPD}=5 \mathrm{~ns})$.

It can be observed that the outage probability can be improved as the block length increases; this is simply due to the fact that an increased block length has more channel realizations and thus leads the block capacity to approach the ergodic capacity limit. Moreover, as the transistor width or the WPD increases, the decreased outage probability should be attributed to both improved operation channel quality and lower write operation failure rate. Note that for large outage probabilities the gain obtained with an increased block length is usually smaller than the gain obtained with improved technical parameters; this is because the former only induces that the block capacity approaches the ergodic capacity while channel conditions are not improved, however, improving technical (i.e., physical) parameters directly increases the channel capacity.

Obviously, there is a price for improved technical parameters. For example, increasing the transistor width does improve the channel capacity and thus allows the use of higher code rate ECCs; however, both the memory area and power consumptionthenincrease.Therefore, for a specific application, 
the optimum solution will be selected by balancing the various requirements among latency, throughput, size, andpower constrain.

\section{CONCLUSION}

This paper proposed a complete channel model to simulate write and read operations of the 1T-1MTJ STT-MRAM cells. This model considered both process variations and thermal fluctuations. Based on the proposed cell operation channel, reliabilities including operation failure rate, bit error rate (BER), channel ergodic capacity and channel outage probabilitywere evaluated from an information theory perspective. Moreover, it is proved that the distributions of the WIB states are not equiprobable and that their ratio isdetermined by their respective write success probabilities. Finally, simulation results show that practical code rates and code block lengths can guarantee reliable performances only if the difference between state ' 1 ' and state ' 0 ' operation success rates is small enough.

\section{REFERENCES}

[1] S. Lin and D. J. Costello, "Error Control Coding," 2nd Edition, Prentice Hall, 2004.

[2] C. Vanstraceele, B. Geller, J.P. Barbot, J.M. Brossier, "A Low Complexity Block Turbo Decoder Architecture," IEEE Trans. on Commun., vol. 56, no 12, pp. 1985-1989, Dec. 2008.

[3] C. Shannon "A Mathematical Theory of Communication," Bell Syst. Technical Journal, vol 27, pp 379-423, 623-656, July-October 1948.

[4] B. Chen, Z. Qin, and K.Cai,"Channel capacity and soft-decision decoding of LDPC codes for spin-torque transfer magnetic random access memory (STT-MRAM)," Intern. Conf. on Computing, Networking and Communications 2013 (ICNC 2013) San Diego, CA, USA, Jan. 28-31, 2013.

[5] W. Wen, M. Mao, X. Zhu, S.H. Kang, "CD-ECC: Content-dependent error correction codes for combating asymmetric nonvolatile memory operation errors," 2013 IEEE/ACM Intern. Conf. on Computer-Aided Design (ICCAD), San Jose, CA, 18-21 Nov. 2013.

[6] X. Fong, S. H. Choday, K. Roy, "Bit-cell level optimization for non-volatile memories using magnetic tunnel junctions and spin-transfer torque switching," IEEE Trans. Nanotechnology, vol. 11, iss. 1, pp. 172-181, Jan. 2012.

[7] W.S. Zhao, Y. Zhang, T. Devolder, J-O. Klein, D. Ravelosona, C. Chappert, P. Mazoyer, "Failure and reliability analysis of STT-MRAM," Microelectronics Reliability, Vol. 52, pp.1848-1852, Jul. 2012.

[8] H. Wang, K. Zhao, J. Li, and T. Zhang, "Optimizing the Use of STT-RAM in SSDs through Data-Dependent Error Tolerance," IEEE Trans. on VLSI Systems, accepted.

[9] C. Yang, Y.Emre, Y. Cao, C.Chakrabarti,"Improving reliability of non-volatile memory technologies through circuit level techniques and error control coding,"EURASIP Jour. on Advances in Signal Proc., pp.1-24, 2012.

[10] W. Kang, W. Zhao, Z. Wang, Y. Zhang, J-O Klein, Y. Zhang, C. Chappert, D. Ravelosona,"A low-cost built-in error correction circuit design for STT-MRAM reliability improvement," Microelectronics Reliability. pp. 1224-1229, 53(9), 2013.

[11] H. Sun, C. Liu, T. Min, N. Zheng, and T. Zhang, "Architectural Exploration to Enable Sufficient MTJ Device Write Margin for MRAM-based Cache," IEEE Trans. on Magnetics, pp. 2346-2351, vol. 48, iss. 8, 2012.

[12] W. Xu, X. Wang, Y. Chen, and T. Zhang, "Improving STT MRAM Storage Density through Smaller-Than-Worst-Case Transistor Sizing," Design Automation Conference (DAC), July 2009.

[13] B. Geller, I. Diatta, J.P. Barbot, C. Vanstraceele,F. Rambeau, "Block Turbo Codes: From Architecture to Application," in Proc. of IEEE International Symposium on Information Theory, ISIT 2006, Seattle, pp. 1813-1816, July 2006.

[14] J. Z. Sun, "Spin-current interaction with a monodomain magnetic body: A model study," Physical Review B, 62,pp. 570-578, 2000.
[15] W.Zhao, E.Belhaire, Q.Mistral, C.Chappert, V.Javerilac, B.Dieny and E.Nicolle "Macro-model of Spin-Transfer Torque based Magnetic Tunnel Junction device for hybrid Magnetic-CMOS design", IEEE BMAS, pp. 40-44. 2006, USA.

[16] J. D. Harms, F. Ebrahimi, Y. Xiaofeng, and W. Jian-Ping, "SPICE macromodel of spin-torque-transfer-operated magnetic tunnel junctions," IEEE Trans. Electron Devices, vol. 57, no. 6, pp. 1425-1430, Jun. 2010.

[17] W. Guo, G. Prenat, V. Javerliac, M. El Baraji, N. de Mestier, C. Baraduc, and B. Diény, "SPICE modelling of magnetic tunnel junctions written by spin-transfer torque," J. Phys. D, Appl. Phys., vol. 43, no. 21, pp. 215001-1-215001-8, Jun. 2010.

[18] L.-B. Faber, W. Zhao, J.-O. Klein, T. Devolder, and C. Chappert, "Dynamic compact model of spin-transfer torque based magnetic tunnel junction (MTJ)," in Proc. 4th Int. Conf. DTIS, Apr. 2009, pp. 130-135.

[19] A. Fert, "Historical Overview: From Electron Transport in Magnetic Materials to Spintronics," Handbook of Spin Transport and Magnetism, pp.3-17, CRC Press, 2012.

[20] L. Berger, "Emission of Spin waves by a magnetic multilayer traversed by a current," Physical Review B, 54, pp. 9353-9358, Oct., 1996.

[21] Y.Ye, F. Liu, M. Chen, S. Nassif, "Statistical Modeling and Simulation of Threshold Variation Under Random Dopant Fluctuations and Line-Edge Roughness," IEEE Trans. on Very Large Scale Integration (VLSI) Systems, pp.987-996, vol.19, June, 2011.

[22] J. Li, C. Augustine, S. Sayeef, K. Roy, "Modeling of failure probability and statistical design of Spin-Torque Transfer Magnetic Random Access Memory (STT MRAM) array for yield enhancement," in Proc. of 45th ACM/IEEE Design Automation Conf., 2008. DAC 2008, pp. 278 - 283, Anaheim, CA, June 8-13, 2008.

[23] X. Wang, Y. Zheng, H. Xi, and D. Dimitrov. "Thermal Fluctuation Effects on Spin Torque Induced Switching: Mean and Variations," Journal of Applied Physics, 103(3), Feb. 2008.

[24] Z. Diao, Z. Li, S. Wang, Y. Ding, A. Panchula, E. Chen, L. Wang, and Y. Huai, "Spin-transfer torque switching in magnetic tunnel junctions and spin transfer torque random access memory," J. Phys., Condensed Matter, vol. 19, no. 16, pp. 165209-1-165209-13, Apr. 2007.

[25] Y. Zhang, X. Wang, and Y. Chen. "STT-RAM Cell Design Optimization for Persistent and Non-Persistent Error rate Reduction: A statistical Design View," in Proc. of IEEE/ACM Intern. Conf. on Computer-Aided Design (ICCAD) 2011, pp. 471-477, San Jose, CA, Nov. 7-10, 2011.

[26] Y. Zhang, X. Wang, Y. Li, A. K. Jones, and Y. Chen. "Asymmetry of MTJ switching and its implication to STT-RAM designs," in Proc. of Design, Automation \& Test in Europe Conference \& Exhibition (DATE) 2012, pp. 1313-1318, Dresden , 2012.

[27] Y. Zhang, W. Wen, and Y. Chen. "STT-RAM Cell Design Considering MTJ Asymmetric Switching," SPIN, 02(03):1240007, 2012.

[28] Y. Zhang, I. Bayram, Y. Wang, H. Li and Y. Chen, "ADAMS: Asymmetric Differential STT-RAM Cell Structure for Reliable and High-performance Applications," in Proc. of IEEE/ACM Conf. on Computer Aided Design (ICCAD) 2013, pp. 9-16, San Jose, CA, Nov. 18-21, 2013.

[29] K. L. Du and M. N. S. Swamy, "Wireless Communication Systems,“ Cambridge University Press, April, 2010.

[30] Y. Zhang, W. Wen, and Y. Chen, "Asymmetry in STT-RAM Cell Operations," Emerging Memory Technologies - Design, Architecture, and Applications, pp.117-144, Springer, 2014.

[31] Predictive Technology Model (PTM). http://www.eas.asu.edu/ ptm/

[32] W. Kang, Z. Li, J.-O. Klein, Y. Chen, Y. Zhang, D. Ravelosona, C. Chappert, and W. Zhao, "Variation-Tolerant and Disturbance-Free Sensing Circuit for Deep Nanometer STT-MRAM," IEEE Trans. Nanotech., 1088-1092, vol.13, iss.6, Sept., 2014.

[33] X. Wang, Y. Chen, H. Li, D. Dimitrov, and H. Liu, "Spin Torque Random Access Memory Down to 22nm Technology," IEEE Trans. on Magnetics, vol. 44, no. 11, pp. 2479-2482, Nov. 2008.

[34] W. Xu, T. Zhang, and Y. Chen, "Design of Spin-Torque Transfer Magnetoresistive RAM and CAM/TCAM with High Sensing and Search Speed," IEEE Trans. on VLSI Systems, pp. 66-74, vol.18, iss.1, Mars, 2009.

[35] C.-T. Cheng, Y.-C. Tsai, and K.-H. Cheng, "A high-speed current mode sense amplifier for Spin-Torque Transfer Magnetic Random Access Memory," in proc. of 53rd IEEE Intern. Midwest Symp. on Circuits and Systems (MWSCAS) 2010, pp. 181-184, Aug. 2010. 


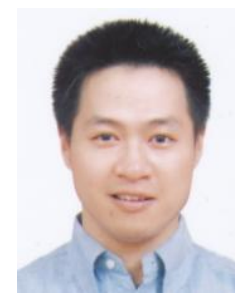

Jianxiao YANG received a $\mathrm{PhD}$ degree from Zhejiang University, China in 2007 on Information and Communication Engineering.

$\mathrm{He}$ joined the research Lab SATIE of the EcoleNormaleSuperieure de Cachan (2007-2009) and the Lab-STICC of Telecom Bretagne (2009-2013). He is currently working as a researcher at Lab U2IS, ENSTA ParisTech since 2013.

His research interests cover digital circuit design for storage systems and wireless sensor networks (IoT).

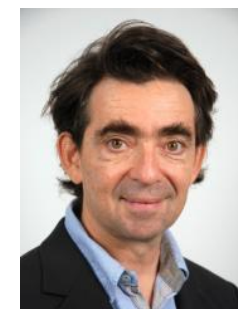

Benoit GELLER received the $\mathrm{PhD}$ in Telecommunications from the INP Grenoble in 1992.

He was the head of the Multisensor and Information Team (TIM, 17 permanent researchers) at SATIE lab - ENS Cachan until he joined ENSTA ParisTech in 2007 where he is currently a Full Professor.

He works on iterative methods with application to digital communications, the Internet of Things and storage networks. He also has a strong experience of coding and wireless networks. information theory, signal processing,error control

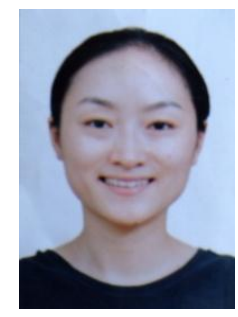

Meng LI received a $\mathrm{PhD}$ degree in electronics engineering from Telecom Bretagne, France in 2011.

She joined the Green Radio Group of IMEC in 2012 and is a research engineer.

Her research interests cover high speed digital circuit design for essential components in base band, especially with the design of decoder for error control codes.

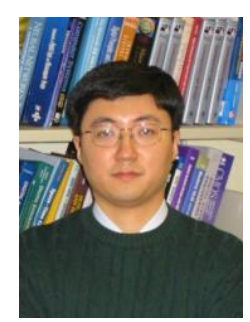

Tong ZHANGreceived the Ph.D. degree in electrical engineering from the University of Minnesota, Minneapolis, in 2002.

$\mathrm{He}$ is currently an Associate Professor with the Department of Electrical, Computer, and Systems Engineering, Rensselaer Polytechnic Institute, Troy, NY.

His research activities span over circuits and systems for various data-storage and computing applications. He currently serves as an Associate Editor of the IEEE Trans. on Circuits and

Systems-II and the IEEE Trans. on Signal Processing. 